\title{
Physiological and physical adaptations within the working muscle specific to cardiovascular training, lactate training, adenosine triphosphate- phosphocreatine training and power training
}

\begin{abstract}
Objectives: The purpose of the present review study is to review the existing literature regarding the physiological and physical adaptations within working skeletal muscle specific to cardiovascular training, lactate training, adenosine triphosphatephosphocreatine training and power training.

Methods: A search was conducted on the wide-body of research that exists in and around the skeletal muscle and sports performance and aligns the research in a clear manner, specifically describing the physiological response of various training to cellular muscle. Literature gathered involved trails of comparative analysis with control groups in various exercise settings.

Results: In an attempt to clarify the physiological adaptations to working skeletal muscle, the purpose of this review is to determine what affect 4 different types of conditioning reflects in terms of structural, metabolic, enzymatic, neuromuscular and contractile. The present study identifies each adaptation specific to the training modality to clarify the scientific evidence for the sport practitioner
\end{abstract}

Keywords: muscle adaptations, adenosine triphosphate, cardiovascular training, power training
Volume I Issue 4 - 2017

\section{Christopher K Carroll}

Department of Human Kinetics and Applied Health Science, Bethel University, USA

\section{Correspondence: Christopher K Carroll, Department of Human Kinetics and Applied Health Science, Bethel University, USA,Email carchrk@bethel.edu}

Received: August 08, 2017 | Published: September 25, 2017
Abbreviations: ATP-PC, adensosine triphosphatephosphocreatine; SSC, stretch shortening cycle

\section{Introduction}

The capacity for change within skeletal muscle is immense. Represented by gene expression on a molecular level, the alteration of muscle generally results in an increase or decrease in the amount of muscle proteins. When exercise stress is placed upon the skeletal muscle, the magnitude of adaptation is highly contingent upon plasticity of muscle. Thus, the Principal of Myoplasticity is the specific term that represents the capacity of muscle that is modifiable for adaptation and applies to the ensuing discussion related to muscular training adaptations. ${ }^{1}$

\section{Cardiovascular training}

Cardiovascular training on a structural level elicits a significant increase in activation frequency of motor units and a slight increase in oppositional load against motor units. Quintessential cardiovascular training that presents this stimulus to the muscle is represented in weight bearing settings (i.e., running) as well as non-weight bearing settings (swimming and cycling). ${ }^{1}$

Cardiovascular training results in little to no effect on the crosssectional area of muscle and muscle fibers. However, specific to type I slow-twitch fibers, these fibers may experience slight increases in cross sectional size due to increases glycogen storage as well as oxidative metabolism. ${ }^{2,3}$ The majority of adaptations to skeletal muscle occur within the metabolic realm. The adaptations to muscle under the influence of cardiovascular training produce changes in fiber type, capillary supply, myoglobin content, mitochondrial function and oxidative enzymes. ${ }^{3}$

\section{Fiber type}

Cardiovascular training relies primarily on type I, slow-twitch muscle fibers. In response to the volume and intensity of training, type I fibers have the capability to become larger in cross-sectional area., Fast-twitch fibers are not generally recruited to the same capacity as slow-twitch during cardiovascular training; therefore don't experience similar results in cross sectional area. Similarly, most literature suggests little change in muscle fiber percentage in slow-twitch and fast-twitch fibers; however, changes have been documented in fast-twitch subtypes. Type IIx fast-twitch fibers have called upon less during cardiovascular training, consequently, possess a lower aerobic capacity. Recent literature produced by the HERITAGE study suggests that type IIx fast-twitch fibers may transform to type IIa and it is even speculated they may be capable of transitioning to slowtwitch. However, very minuscule results have been published.

\section{Capillary supply}

A significant adaptation to cardiovascular training is the increase in capillaries surrounding the muscle fibers. Literature has documented that cardiovascular trained individuals have significantly greater amounts of capillary density than compared to sedentary populations. ${ }^{4,5}$ Dense capillary content allows a heightened interchange of gasses, heat, waste and nutrients between the working skeletal muscle and the blood delivered. ${ }^{3}$ 


\section{Myoglobin content}

Upon entering the muscle, oxygen binds with an iron-containing compound that resembles hemoglobin called myoglobin. Myoglobin transports oxygen molecules from the cell membrane to the mitochondria. Of importance to cardiovascular training, myoglobin has the capacity to store oxygen and release it, particularly when oxygen becomes sparse during muscle contraction. The release of oxygen often occurs during the lag time between the beginning of exercise and the amplified cardiovascular transportation of oxygen. Although the detailed allocations of oxygen delivery are not completely understood, cardiovascular training has been documented to increase myoglobin content by $75-80 \%$. This adaption of muscle significantly impacts the ability for oxidative metabolism. ${ }^{3}$

\section{Mitochondrial function}

Aerobic energy production occurs within the mitochondria Therefore the affect on the mitochondrial function is obvious. The major adaptations with cardiovascular training on the mitochondria are increases in size and number. Literature has depicted increases in the actual number of mitochondria by as much as $15 \%$, as well as the total size by as much as $35 \%$ in as little as 27 -weeks of training. As the volume of cardiovascular training rises, so do the number and the size of the mitochondria.

\section{Oxidative enzymes}

Mitochondrial efficiency and the oxidative formation of ATP are increased by oxidative enzymes. Cardiovascular training has been shown to increase these enzymes' activity. Training induced enzyme activity contributes not only to the number and size of the mitochondria, but also to the metabolic consequence of mitochondrial changes. It is suggested that increased enzyme activity creating mitochondrial changes creates a slower use of muscle glycogen and a reduced production of lactate during exercise at given intensities. This adaptation is likely to have great importance to ones lactate threshold.

\section{Energy sources}

Cardiovascular training forces adaptations on the muscle's ability to store and metabolize glycogen and fat. Muscle glycogen is often the primary substrate for cardiovascular exercise. The primary response for depleted glycogen storage is a heighted resynthesis capacity. With proper recover and dietary intake, cardiovascularly trained muscle stores significantly greater amounts of glycogen. ${ }^{7}$ Additionally, cardiovascularly trained muscle contains higher amounts of intermuscular triglycerides. Furthermore, muscular enzymes responsible for lipid breakdown are increased with cardiovascular training. This adaptation allows for trained muscle to oxidize lipids, decreasing the breakdown of glycogen. ${ }^{3}$

\section{Lactate training}

In addition to the cardiovascular adaptations and benefits discussed above, lactate training results in a few other key muscle adaptations. Lactate training places high stress on the glycolytic system within the muscle resulting in high amounts of lactate accumulation. Training under the presence of lactate will lead to increased removal avenues. Increased lactate transporter, monocarboxylate (MCT) and mitochondrial proteins are expressed in greater capacities in response of higher lactate concentrations and heighten the muscles potential for lactate removal. $^{8}$
Intercellular buffer capacity also contributes to the removal of lactate during lactate training. Physicochemical buffering proteins, dipeptides and phosphates within skeletal muscle increase the efficiency of lactate removal when lactate training is performed. Similarly, the local formation of lactate within skeletal muscle is theorized as a potential mechanism for adaptation of muscular $\mathrm{pH}$ regulation. $\mathrm{P}^{\mathrm{H}}$ displacement during lactate training may result in adaptations for improved lactate clearance. ${ }^{8}$

The overall adaptation of lactate training is to increase lactate tolerance of skeletal muscle. Buffering and removal capacities of skeletal muscle allow for increased concentration of muscle and blood lactate. Thus, neutralizing the effect lactate plays on muscle, therefore delaying performance fatigue.

\section{Adenosine triphosphate- phosphocreatine (ATP-PC) training}

\section{Neuromuscular}

One of the most significant adaptations of ATP-PC training that explains much of the gains that are made by the athletes that are trained are neural. Motor unit recruitment and synchronization explain much of the gains that are made during ATP-PC training in the absence of hypertrophy. Connections made between the spinal cord (motor neurons) and the motor units to synchronize contraction leads to the muscle's ability to produce more force. Similarly, the more units recruited to perform muscle actions, lead to maximal contraction

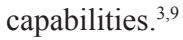

Autogenic inhibitory mechanisms (i.e. GTO's) may gradually be reduced in their capacity to counteract impulses, therefore lead to the potential for greater strength when ATP-PC training is administered. ${ }^{10}$ Adaptations made limiting the effect of the inhibitory mechanisms within the neuromuscular system may create a platform for greater force production.

Co-activation (agonist and antagonist muscles) may be another neural factor to ATP-PC training. Reducing the amount of resistive force of antagonist muscle may contribute to greater force production of agonist muscles. Although speculated to provide minimal contribution, the reduction of co-activation may contribute to neuromuscular adaptations leading to greater force production. ${ }^{11,12}$

Finally, rate coding may be a contributing neuromuscular factor contributing to greater force production succeeding ATP-PC training. The firing frequency of motor units, known as rate coding, may also lead to force generating improvements. Although not well documented, evidence does exist to underpin this ATP-PC training mechanism. ${ }^{11,12}$

\section{Contractile Components}

Hypertrophy: Two types of muscle hypertrophy occur with ATPPC training. The first, Transient lasts only a short time immediately after a training bout and is caused by fluid buildup from blood plasma in the interstitial and intercellular spaces within muscle. Chronic hypertrophy reflects the actual muscle structure size increases that are a result of fiber hypertrophy (increase in existing fiber size) or fiber hyperplasia (increase in actual number of fibers). Current research hasn't completely solidified the debate as to whether or not increases in muscle size are a result of fiber hyperplasia. Early research established that the number of muscle fibers an individual 
possess is genetic and the alteration of muscle size rests solely in fiber hypertrophy. However, a growing amount of animal research suggests hyperplasia is a result of ATP-PC training, but only a few studies represent hyperplasia occurring in humans. ${ }^{3,8}$

Myosin heavy chain II: In addition to cross sectional muscle area increases (hypertrophy),

additional myosin heavy chain II isoforms are present as a result of ATP-PC training. ${ }^{1}$

Type I and Type II fiber area: ATP-PC training also has the ability to alter muscle fiber area.

Heavily trained subjects (depending on training intensity and volume) have experienced major alteration in type II muscle fiber diameter in comparison to type I. ${ }^{1,13,14}$

\section{Capillary and mitochondrial density}

Chronic ATP-PC training can have the ability to affect the aerobic metabolism of type I fibers. As type II fibers increase in crosssectional size, mitochondrial and capillary densities decrease. The absolute mitochondrial and capillary volumes remain unchanged, however, substrate transport, oxygen utilization and carbon dioxide withdraw may be limited. The end result is a theorized decrease in cardiovascular capacity. ${ }^{1,13,14}$

\section{Biochemical}

ATP-PC training of high intensity and slow-speed utilizing Isokinetic loading may increase several biochemical mechanisms. Muscle glycogen content, CP, ATP, ADP, creatine, phosphorylase, phosphofructokinase and Krebs cycle enzyme activity are all speculated to experience increased levels under the presence of ATPPC training. However, ATP-PC training at higher speeds, more related to Power Training, do not replicate the same biochemical results.

\section{Power training}

Power training will elicit similar results as experienced with ATPPC training, particularly the neuromuscular effects. However, in addition to the neural adaptations of muscle with ATP-PC training, power training also stresses the elastic components. Power training, often carried out with the utilization of plyometric loading and emphasizes the stress of the stretch shortening cycle (SSC). More specifically, power training generally involves sudden eccentric stress (the stretch of the muscle) followed by a sudden, rapid fast movement concentric contraction. The results of power training are not only increases in muscle strength but also shortening muscle contractile time. ${ }^{1}$

\section{Neural changes}

Primary neuromuscular adaptations witnessed with power training are shortening the time of motor unit recruitment, particularly of the type IIx and IIa fibers and heightening the tolerance of motor neurons to greater intervention frequencies. ${ }^{15}$ Specifically, power training initiates and escalates the discharge rate of type IIx and IIa muscle fibers increasing synchronization of motor units and the rate of firing. ${ }^{16}$

Power training also activates greater intramuscular coordination. Improved linkage between nerve impulses and skeletal muscle, results in greater neuromuscular coordination of the agonist and antagonist muscles. This adaptation is similar to the discussion above regarding co activation. ${ }^{16}$

\section{Conclusion}

The magnitude of change and adaptation within skeletal muscle is vast. Largely contingent on the stress placed on upon the body from a training standpoint, muscle will respond and adapt differently, based on the type and modality of exercise. As described above, the most significant training adaptations from cardiovascular training are found within the muscle's capillary supply, myoglobin content, mitochondrial function and enzymatic efficiency. These adaptations are the major players for improved performance. Specifically, capillary content creates improved interchange of gasses, heat, waste and nutrients between the working skeletal muscle and the blood delivered during exercise. Increased myoglobin content has the capacity to store oxygen and release it, particularly when oxygen becomes sparse during muscle contraction during prolonged cardiovascular exercise. Given aerobic energy production occurs within the mitochondria, the improvement of its function, particularly in size and number, becomes an obvious player in improved cardiovascular performance. Lastly, from a cardiovascular standpoint, the efficiency of enzymatic activity is demonstrated to create mitochondrial changes which therefore serves as a vehicle for slower use of muscle glycogen and a reduced production of lactate during exercise at given intensities. This adaptation likely serves as a major prayer in a heighted lactate threshold.

Lactate training, on the other hand, increases the muscle's capacity to tolerate inorganic and lactate, primarily by increasing the removal and buffering capacities. This neutralizing muscle adaption limits effect lactate has on skeletal muscle, therefore delaying performance fatigue. Training within the energy system of the ATP-PC system, results in primarily neuromuscular and contractile component adaptations such as hypertrophy and co-coordination of the muscles. Connections made between the spinal cord (motor neurons) and the motor units to synchronize contraction leads to the muscle's ability to produce more force. Autogenic inhibitory mechanisms (i.e. GTO's and Muscle Spindles) may progressively be diminished in their capacity to counteract impulses, therefore lead to the potential for greater force production. Finally co-activation (agonist and antagonist muscles) improvements by reducing the amount of resistive force of antagonist muscle, is supported to contribute to greater force production of agonist muscles.

Similarly, power training results in significant neuromuscular adaptations, however, the elasticity of muscle, specifically the SSC, is more pronounced within power training. Given that most power training involves sudden eccentric stress (the stretch of the muscle) followed by a sudden, rapid fast movement concentric contraction, chronic power training may lead to increases in muscle strength but also shortening muscle contractile time. Training for power also shortens the time of motor unit recruitment, particularly of the type IIx and IIa fibers, therefore heightening the tolerance of motor neurons to improve intervention frequencies. Specifically, a more expedited initiation and excitement of the discharge rate of type IIx and IIa muscle fibers will in turn increase the synchronization of motor units and the rate of firing. The plasticity of muscle is highly contingent on the stress placed upon it and the adaptions are extensively different and described above. 


\section{Acknowledgement}

None.

\section{Conflict of interest}

Author declares there is no conflict of interest in publishing the article.

\section{References}

1. Brooks GA, Fahey TD, Baldwin KM. Exercise physiology: Human bioenergetics and its applications, 4th edition. McGraw Hill, USA; 2005.

2. Sanz RJ, Rankinen T, Joanisse DR, et al. Familial resemblance for muscle phenotypes in the HERITAGE family study. Med Sci Sports Exerc. 2003;35(8):1360-1366.

3. Wilmore JH, Costill DL. Physiology of sport and exercise, 3rd edition. Human Kinetics, USA; 2004.

4. Lewis MI, Fournier M, Wang H, et al. Effect of endurance and/or strength training on muscle fiber size, oxidative capacity and capillarity in hemodialysis patients. J Appl Physiol. 2015;119(9):865-871.

5. Hermansen L, Wachtlova M. Capillary density of skeletal muscle in welltrained and untrained men. J Appl Physiol. 1971;30(6):860-863.

6. Holloszy JO, Coyle EF. Adaptations of skeletal muscle to endurance exercise and their metabolic consequences. J Appl Physiol Respir Environ Exerc Physiol. 1984;56(4):831-838.

7. Greiwe JS, Hickner RC, Hansen PA, et al. Effects of endurance exercise training on muscle glycogen accumulation in humans. J Appl Physiol. 1999;87(1):222-226.
8. Juel C. Muscle pH regulation: role of training. Acta Physiol Scand. 1998;162(3):359-366.

9. Hawley JA, Hargreaves M, Joyner MJ, et al. Integrative Biology of Exercsie. Cell. 2014;159(4):738-749.

10. Aagaard P, Simonsen EB, Andersen JL, et al. Neural inhibition during maximal eccentric and concentric quadriceps contraction: Effects of resistance training. J Appl Physiol. 2000;89(6):2249-2257.

11. Gee MSL, Swinton C, Morrison S, et al. Compensatory regulation of HDAC5 in muscle maintains metabolic adaptive responses and metabolism in response to energetic stress. 2014;28(8):3384-3395.

12. Enoka RM. Neural adaptations with chronic physical activity. $J$ Biomech 1997;30(5):447-455

13. Geng T, Li P, Okutsu M, et al. PGC-1 $\alpha$ plays a functional role in exerciseinduced mitochondrial biogenesis and angiogenesis but not fiber-type transformation in mouse skeletal muscle. Am J Physiol Cell Physiol. 2010;298(3):C572-C579.

14. Rowe GC, Khoury R, Patten IS, et al. PGC-1 $\alpha$ is dispensible for exercise induced mitochondrial biogenesis in skeletal muscle. PLoS One. 2012;7(7):e41817.

15. Hakkinen K. Neuromuscular and hormonal adaptations during strength and power training. A review. J Sports Med Phys Fitness. 1989;29(1):9-26.

16. Bompa TO, Carrera MC. Periodization training for sports, 2nd edition. Human Kinetics, USA; 2005. 